\title{
Nanocomposites Based on Magnesium-oxide/ Aluminum-Nitride/Polypropylene for HVDC Cable insulation
}

\author{
Xiaosi Lin \\ Department of Electronic and Electrical \\ Engineering \\ University of Strathclyde \\ Glasgow, United Kingdom \\ xiaosi.lin@strath.ac.uk \\ John Liggat \\ Department of Pure and Applied \\ Chemistry \\ University of Strathclyde \\ Glasgow, United Kingdom \\ j.j.liggat@strath.ac.uk
}

\author{
WH.Siew \\ Department of Electronic and Electrical \\ Engineering \\ University of Strathclyde \\ Glasgow, United Kingdom \\ wh.siew@strath.ac.uk \\ Jinliang $\mathrm{He}$ \\ Department of Electrical Engineering \\ Tsinghua University \\ Beijing, China \\ hej1@tsinghua.edu.cn
}

\author{
Martin Given \\ Department of Electronic and Electrical \\ Engineering \\ University of Strathclyde \\ Glasgow, United Kingdom \\ m.given@strath.ac.uk
}

\begin{abstract}
Polypropylene (PP) with high thermal stability and good electrical properties, has attracted much attention for its potential to take the place of cross-link polyethylene (XLPE) as HVDC insulation because PP is more easily recycled than XLPE due to its thermoplasticity. Due to the adverse effect of electric field reversal under HVDC application, there is a need to find the new polymer insulation material with higher thermal conductivity and good electrical performance. This paper investigates the effect of introducing aluminum nitride (AIN) and magnesium oxide (MgO) into PP on the electrical properties of the resulting the new nanocomposites. In the sample preparation, $\mathrm{AIN}$ and $\mathrm{MgO}$ were surface-modified by $\mathrm{KH570}(\gamma$ methacryloxypropyltrimethoxy silane) and then introduced into PP by the solution method to manufacture the nanocomposite materials. The measurements made were the voltage breakdown characteristics and the DC conductivity. The results obtained show that the combination of $\mathrm{AIN}$ and $\mathrm{MgO}$ can slightly decrease the DC conductivity of PP/AIN/MgO nanocomposites compared with pure PP. The breakdown strength was slightly decreased. which shows that the adverse effect of AIN on the electrical performance of $P P$ can be compensated by introducing $\mathrm{MgO}$ nanoparticles. Hence, the new polymer with high thermal conductivity and good electrical properties could be manufactured by combining two kinds of nanoparticles.
\end{abstract}

Keywords - nanocomposites, magnesium-oxide, aluminumnitride, polypropylene, electrical performance.

\section{INTRODUCTION}

Polymers filled with nanoparticles have attracted a large amount of attention in recent years since the first concept was proposed by Lewis in 1994 [1]. Due to the special characteristics of nanoparticles, the electrical, chemical, mechanical and thermal properties of polymers can be greatly improved by embedding nanoparticles [2-4]. For example, the space charge accumulation can be suppressed in polypropylene (PP) by introducing nano-MgO nanoparticles. The interfacial region between nanoparticles and polymer determines the characteristics of nanocomposites [6-7].

Polypropylene (PP) is one of the commercial thermoplastic polymers which has larger potential to be the next generation of new polymer insulation material.
Compared with LDPE and XLPE, PP has much higher operational temperature, excellent electrical properties including breakdown strength, space charge characteristics, low DC conductivity and low dielectric loss [8]. The nano$\mathrm{MgO}$ can increase the breakdown strength and decrease the space charge accumulation but the thermal conductivity it has is only $42 \mathrm{~W} \cdot \mathrm{m}^{-1} \cdot \mathrm{K}^{-1}$, while nano-AlN has the thermal conductivity of $275 \mathrm{~W} \cdot \mathrm{m}^{-1} \cdot \mathrm{K}^{-1}$ which is much higher than nano-MgO so that it can increase the thermal conductivity of polymer insulation materials then the electric field distribution would be more uniform and the effect of electric field reversal can be reduced. [9]. Also, both of nano- $\mathrm{MgO}$ and nano-AlN can reduce the dielectric loss of polymer insulation materials.

In this paper, PP was chosen to be the matrix material. Nano-MgO and nano-AlN were introduced into PP with different contents by using the solution method. The objective is to investigate whether it is possible to produce a nanocomposite that possesses the advantages of both additives. Finally, the properties of these nanocomposites were evaluated by DC conductivity and breakdown strength and the results showed that the synergistic effect of nano-AIN and nano-MgO on PP was good.

\section{EXPERIMENTS}

\section{A. Materials and Preparation}

The matrix material, polypropylene (PP) pellets with density of $0.92 \mathrm{~g} / \mathrm{cm}^{3}$ and nanoparticles $\mathrm{MgO}$ and $\mathrm{AlN}$ with a nominal diameter of $50 \mathrm{~nm}$ were provided by Aladdin Industrial Inc., China. The melt flow index of PP was $0.6 \mathrm{~g} / \mathrm{min}$. The xylene solution with a purity of $\geq 98 \%$ was provided by Sinopharm Chemical Reagent. Co. Ltd (China). The nanoparticles of $\mathrm{MgO}$ and $\mathrm{AlN}$ were surface modified by $\gamma$-methacryloxypropyltrimethoxysilane (MPS) in the xylene to improve the adhesion between the nanoparticles and polymer matrix [5]. Normally, it is widely believed that the xylene was used to dissolve the PP pellets at $120^{\circ} \mathrm{C}$. The different contents of nanoparticles shown in Tab.I. were 
dispersed in the mixture of PP and xylene and then the mixed solution was stirred by the blender as shown in Figure 1. The rotor speed was $500 \mathrm{rpm}$, the mixing time was $12 \mathrm{~h}$ and the operational temperature was $120^{\circ} \mathrm{C}$. Next, the mixed solution was dried in the turbo blower at $120{ }^{\circ} \mathrm{C}$ for $12 h$. The film samples with different thickness were manufactured by using compression

TABLE I. SAMPLE NOTATION AND COMPOSITION

\begin{tabular}{|c|c|c|c|}
\hline \multirow{2}{*}{} & \multicolumn{3}{|c|}{ Contents of nano-MgO, nano-AIN and PP } \\
\cline { 2 - 4 } & $\boldsymbol{M g O}(\boldsymbol{w t} . \%)$ & $\boldsymbol{A l N}(\boldsymbol{w t} . \%)$ & $\boldsymbol{P P}(\boldsymbol{w t} . \%)$ \\
\hline No.1 & 0 & 0 & 100 \\
\hline No.2 & 1.0 & 2.0 & 97.0 \\
\hline No.3 & 2.0 & 1.0 & 97.0 \\
\hline No.4 & 3.0 & 0 & 97.0 \\
\hline
\end{tabular}

molding at the temperature of $200{ }^{\circ} \mathrm{C}$ and the duration was 12 min under a pressure of $15 \mathrm{MPa}$. After that, the films were cooled to room temperature under a pressure of $10 \mathrm{Mpa}$ for $15 \mathrm{~min}$. The thickness of the samples was about $100 \mathrm{um}$ for the breakdown test. Finally, the sample of pure PP and nanocomposites with different content of $\mathrm{MgO} / \mathrm{AlN}$ were put in a vacuum oven $(0.01 \mathrm{~Pa})$ at $80{ }^{\circ} \mathrm{C}$ for $24 \mathrm{~h}$ and cooled down to room temperature at $0.1 \mathrm{~K} / \mathrm{min}$.

\section{B. Detection Of Nanoparticles Dispersion}

The nanoparticle dispersion was detected by the field emission scanning electron microscope (FE-SEM, Hitachi SU8010, Japan). The sample of nanocomposites were broken into serval parts in liquid nitrogen, then covered with gold on the fractured surface.

\section{DC Breakdown Tests}

The DC breakdown strength was carried out using a sphere-sphere electrode configuration. The nanocomposite samples with the thickness of 100 um were put into silicone oil so that the nanocomposite samples were isolated from the air and flashover can be avoid during the breakdown test. There are 19 measuring points being marked uniformly on each film and placed in the middle of the sphere electrodes to make the breakdown points happen at the point which has been measured during DC breakdown test. The increasing rate of DC voltage was $1 \mathrm{kV} / \mathrm{s}$ until breakdown occurred.

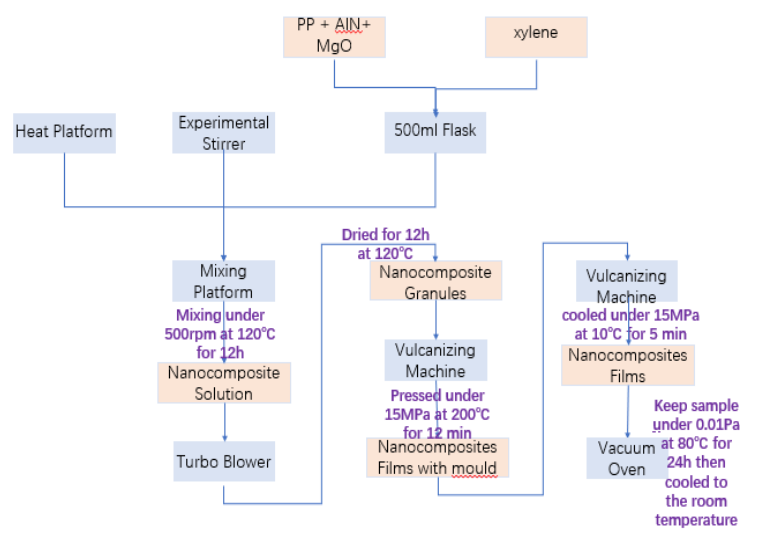

Fig.1. The procedure of sample preparation

\section{DC Conductivity}

The DC volume conductivity was carried out by a digital high-resolution electrometer $(6517 \mathrm{~B}$, Keithley) equipped with standard three-electrodes system. The PP/MgO/AlN nanocomposites samples with the thickness of $100 \mathrm{um}$ were measured under the DC electric field from $10 \mathrm{kV} / \mathrm{mm}$ to $80 \mathrm{kV} / \mathrm{mm}$ with the step of $10 \mathrm{kV} / \mathrm{mm}$. The duration of each step was $600 \mathrm{~s}$. Before the sample measurement, each sample should be short-circuited for $10 \mathrm{~min}$. Finally, the conductivity results will show the effect of nano-MgO and nano-AlN on the DC volume resistivity of PP.

\section{RESULTS AND DISCUSSION}

\section{A. Scanning Electron Microscope Result}

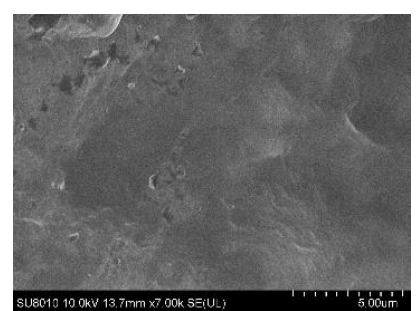

(a)

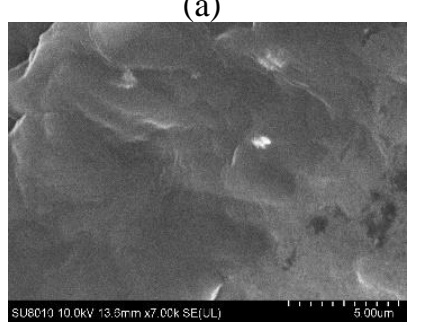

(c)

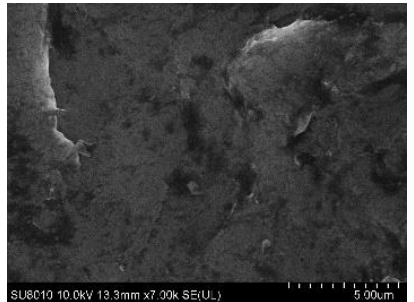

(b)

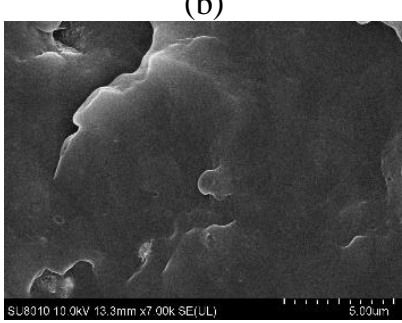

(d)

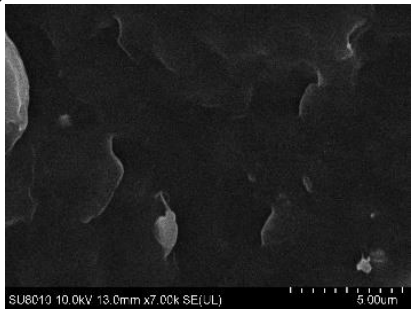

(e)

Fig. 2 SEM images of $\mathrm{MgO} / \mathrm{AlN} / \mathrm{PP}$ nanocomposites with different content (a). Pure PP (b). PP/1.0 phr MgO + 2phr AlN (c). PP/1.0 phr MgO + 2.0 phr AlN (d). PP/3.0 phr MgO (e). PP/3.0 phr AlN

Fig.2 has shown the distribution of nano-MgO and nanoAlN particles in the bulk of the matrix material, PP. Normally the dispersion of nanoparticles has great impact on the electrical properties of nanocomposites. According to the SEM images Fig.2(a)-(e), the surface modification by $\gamma-$ methacryloxypropyltrimethoxysilane (MPS) has ensured most of nano-MgO and nano-AlN particles homogenously dispersed in the matrix material which reveals that the interface between the surface of nano- $\mathrm{MgO}$ and nano- $\mathrm{AlN}$ is compatible and the agglomeration would not cause severe degradation of electrical properties of $\mathrm{MgO} / \mathrm{AlN} / \mathrm{PP}$ nanocomposites.

\section{B. DC Breakdown Tests Result}

The experimental DC breakdown strength of nanocomposites are changeable, hence, the probability 
distribution used to describe the variability was the 2parameter cumulative Weibull distribution function (1) [10]:

$$
P(E)=1-\exp \left[-\left(\frac{E}{E_{0}}\right)^{\beta}\right]
$$

Where:

E : the measured electric field for each measurement point;

$\mathrm{E}_{0}$ : the critical parameter of the breakdown for a probability of $63.2 \%$;

$\beta$ : the shape parameter of the Weibull distribution of breakdown strength data;

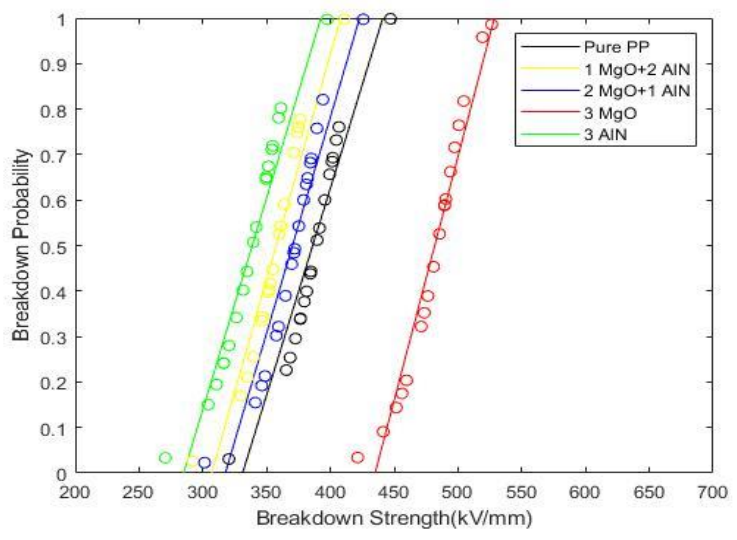

Fig. 3. Weibull description of DC breakdown strength of $\mathrm{PP} / \mathrm{MgO} / \mathrm{AlN}$ nanocomposites

Fig. 3 and Tab. II shows the DC breakdown strength of $\mathrm{PP} / \mathrm{MgO} / \mathrm{AlN}$ nanocomposites with different contents of nano-MgO and nano-AlN. The surface of nano-AlN has been modified by MPS silane coupling agent to achieve good adhesion between nanoparticle and the matrix material PP, but some disadvantage of decreasing the electrical performance would be introduced into the polymer [11]. The nanocomposites with $3 \mathrm{wt} \% \mathrm{MgO}$ had the highest breakdown strength of $492.2 \mathrm{kV}$, while the breakdown strength of nanocomposites with $3 \mathrm{wt} \%$ AlN was reduced to $348.4 \mathrm{kV}$ by $12.3 \%$ compared with PP. The nanocomposites with $2 \mathrm{wt} \%$ $\mathrm{AlN} / 1 \mathrm{wt} \% \mathrm{MgO}$ and $1 \mathrm{wt} \% \mathrm{AlN} / 2 \mathrm{wt} \% \mathrm{MgO}$ had only $7.8 \%$ and $4.17 \%$ lower than the breakdown strength of PP respectively. Thus, the adverse effect of nano-AlN on breakdown strength had been significantly compensated by nano-MgO. It is obvious that the DC breakdown strength of $\mathrm{PP} / \mathrm{MgO} / \mathrm{AlN}$ nanocomposites were reduced due to a higher AlN content. The reduction of DC breakdown strength may be caused by the defects in the interphase between AlN and PP [9].

TABLE II. THE CRITICAL VALUES OF THE BREAKDOWN STRENGTH FOR THE PROBABILITY OF $63.2 \%$

\begin{tabular}{|c|c|c|c|c|c|}
\hline \multirow{2}{*}{} & \multicolumn{5}{|c|}{ Order of Samples } \\
\cline { 2 - 6 } & $\mathbf{1}$ & $\mathbf{2}$ & $\mathbf{3}$ & $\mathbf{4}$ & $\mathbf{5}$ \\
\hline $\mathrm{E}_{0}(\mathrm{kV} / \mathrm{mm})$ & 397.7 & 366.6 & 381.1 & 492.2 & 348.4 \\
\hline
\end{tabular}

\section{DC Conductive Current}

Through the applied DC electric field from $10 \mathrm{kV} / \mathrm{mm}$ to $80 \mathrm{kV} / \mathrm{mm}$ with the step of $10 \mathrm{kV} / \mathrm{mm}$, the conductive current- voltage (J-E) characteristics of all nanocomposites are shown in Fig.4. The DC conductive current increases with the increase of nano-AlN filler content. The nano-MgO could significantly suppress the conductive current. Hence, the increased DC conductivity should be attributed to the higher conductivity of AlN nanoparticles compared with the DC conductivity of $\mathrm{PP}$ and $\mathrm{MgO}$. Otherwise, the low conductivity of $\mathrm{MgO}$ nanoparticles increases the resistivity of sample. Also, Fig.4. shows that the transition field decrease from $50 \mathrm{kV} / \mathrm{mm}$ to $30 \mathrm{kV} / \mathrm{mm}$ with the increase of nano-AlN. From [12], nano- $\mathrm{MgO}$ can significantly increase the depth of electron trap in PP, which can suppress the electron mobility in the nanocomposites. Hence, the adverse effect of nanoAlN on the conductive current can be compensated by introducing nano- $\mathrm{MgO}$. This phenomenon is presumed to be related to the conductivity of AlN. This phenomenon is presumed to be related to the lower conductivity of nano-AIN than nano-MgO.

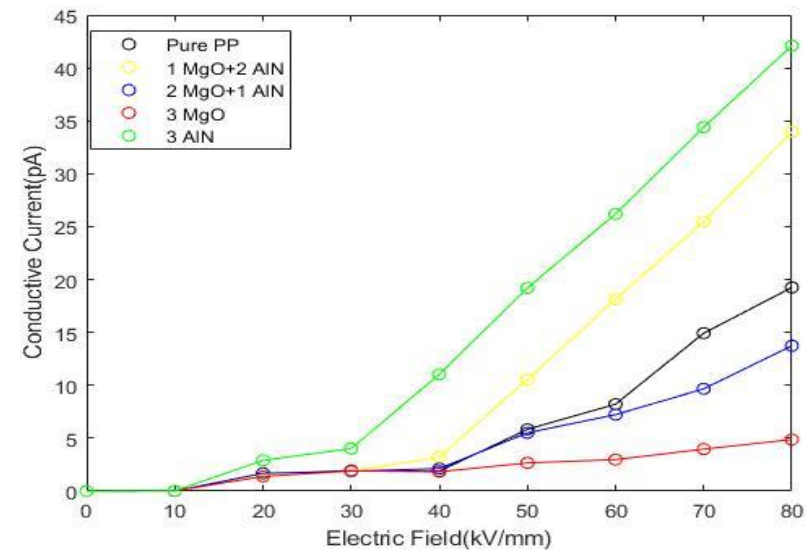

Fig.4. The conductive current under different electric field for all nanocomposites

\section{CONCLUSION}

The addition of nano-AlN and nano-MgO modified with $\gamma$-methacryloxypropyltrimethoxysilane (MPS) have significant effect on the electrical properties on PP. The electrical properties would be worsened by introducing nano$\mathrm{AlN}$, while the nano-MgO could improve the electrical properties of nanocomposite. Even though nano-AlN could worsen the electrical properties of nanocomposites, introducing nano-MgO have significantly enhanced the breakdown strength and reduce conductivity of the nanocomposite. Also, there was not the rapid degradation of electrical performance which means the compatibility between nano- $\mathrm{MgO}$ and nano-AlN in terms of electrical performance was good and the interface relation between nano-AlN and nano-MgO does not have additional effect on electrical application under HVDC condition. Although, the breakdown strength and conductivity performance of the $\mathrm{PP} / \mathrm{MgO} / \mathrm{AlN}$ nanocomposite was reduced, this new nanocomposite still has good electrical properties and higher thermal conductivity, which means the effect electric field reversal on the HVDC application of the PP/MgO/AlN nanocomposites has been reduced. The results indicate that a nano-composite embedded with two types of nanoparticles complement each other and further investigation into the 
other properties (chemical and electrical) would be done to fully assess the viability of this approach.

In the future work, more tests including thermal conductivity measurement, pulse electro-acoustic (PEA) test and dielectric characteristics will be done to do the further research on these nanocomposites' characteristics.

\section{ACKNOWLEDGMENT}

The Authors are very grateful to the State Key Laboratory of Power Systems, Department of Electrical Engineering, Tsinghua University, Beijing for the support of experimental facilities.

\section{REFERENCE}

[1]. T. J. Lewis, "Nanometric Dielectrics," IEEE Trans. Dielectr. Electr. Insul., Vol. 1, No. 5, pp. 812-825, 1994.

[2]. T. Tanaka, M. Kozako, N. Fuse, and Y. Ohki, "Proposal of a multi-core model for polymer nanocomposite dielectrics," IEEE Trans. Dielectr. Electr. Insul., Vol. 12, No.4, pp. 669-681, 2005.

[3]. T. Tanaka, "Dielectric nanocomposites with insulating properties," in IEEE Transactions on Dielectrics and Electrical Insulation, vol. 12, no. 5, pp. 914-928, Oct. 2005.

[4]. T. Tanaka, M. Kozako, N. Fuse, and Y. Ohki, "Proposal of a multicore model for polymer nanocomposite dielectrics," IEEE Trans. Dielectr. Electr. Insul., Vol. 12, No.4, pp. 669-681, 2005.

[5]. Z. Li, W. Cao, G. Sheng, X. Jiang and M. G. Danikas, "Experimental study on space charge and electrical strength of $\mathrm{MgO}$ nanoparticles/polypropylene composite," in IEEE Transactions on Dielectrics and Electrical Insulation, vol. 23, no. 3, pp. 1812-1819, June 2016.

[6]. T. J. Lewis, "Interfaces are the dominant feature of dielectrics at the nanometric level," IEEE Trans. Dielectr. Electr. Insul., Vol. 11, No. 5, pp. 739-753, 2004.

[7]. M. Roy, J. K. Nelson, R. K. Maccrone, L. S. Schadler, C. W. Reed, R. Keefe and W. Zenger, "Polymer nanocomposite dielectrics-The role of the interface," IEEE Trans. Dielectr. Electr. Insul., Vol. 12, No. 4, pp. 629-643, 2005.

[8]. J. Zha, Y. Wu, S. Wang, D. Wu, H. Yan and Z. Dang, "Improvement of space charge suppression of polypropylene for potential application in HVDC cables," in IEEE Transactions on Dielectrics and Electrical Insulation, vol. 23, no. 4, pp. 2337-2343, August 2016.

[9]. Y. Zhou, J. Hu, X. Chen, F. Yu and J. He, "Thermoplastic polypropylene/aluminum nitride nanocomposites with enhanced thermal conductivity and low dielectric loss," in IEEE Transactions on Dielectrics and Electrical Insulation, vol. 23, no. 5, pp. 27682776, October 2016.

[10]. IEC 62539,"Guide for the statistical analysis of electrical insulation breakdown data".

[11]. T. Tanaka, A. Vaughan, "Tailoring of Nanocomposite Dielectrics". Pan Stanford Publishing, 2016.

[12]. Y. Zhou et al., "Temperature dependent electrical properties of thermoplastic polypropylene nanocomposites for HVDC cable insulation," in IEEE Transactions on Dielectrics and Electrical Insulation, vol. 26, no. 5, pp. 1596-1604, Oct. 2019. 